\title{
Nanosilver-Mediated Change in Human Intestinal Microbiota
}

\section{Pranab Das ${ }^{1 *}$, Julie AK McDonald ${ }^{2}$, Elaine O Petrof ${ }^{2}$, Emma Allen-Vercoe ${ }^{3}$ and Virginia K Walker ${ }^{1,4}$}

${ }^{1}$ Department of Biology, Queen's University, Kingston, ON K7L 3N6, Canada

${ }^{2}$ Department of Medicine, Kingston General Hospital, Queen's University, Kingston, ON K7L 2V7, Canada

${ }^{3}$ Department of Molecular and Cellular Biology, University of Guelph, Guelph, ON N1G 2W1, Canada

${ }^{4}$ Department of Biomedical and Molecular Sciences, and School of Environmental Studies, Queen's University, Kingston, ON K7L 3N6, Canada

\begin{abstract}
The widespread use of silver nanoparticles (AgNPs) has prompted studies on different species and at various trophic levels, however, little is known about possible toxicological impacts on their corresponding microbiota. Here the effects of AgNPs on a defined bacterial community established from the collected stool of a healthy donor have been investigated. The anaerobic consortium was exposed to several concentrations $(0-200 \mathrm{mg} / \mathrm{L})$ of AgNPs (capped; 10 $\mathrm{nm}$ ) for $48 \mathrm{~h}$. Nanosilver had a negative impact on the bacterial community as evidenced by a significant reduction $(\sim 22 \%)$ in culture-generated gas production at the two highest AgNP concentrations compared to controls, as well as significant $(p<0.05)$ changes in fatty acid methyl ester profiles even at the lowest concentration. DNA analysis confirmed these observations showing a shift in the community structure as demonstrated by cluster analysis of PCR-denaturing gradient gel electrophoresis (DGGE) profiles, as well by phylogenetic distributions obtained from 454 pyrotag $16 \mathrm{~S}$ rRNA gene sequencing. The bacterial assemblage was significantly $(p<0.01)$ different in the AgNP- treatment groups compared to controls. As expected, ionic silver ( $\mathrm{AgCl} ; 25-200 \mathrm{mg} / \mathrm{L})$ was also antibacterial, as shown by all four assessment methods, but its effects were distinct from the AgNP-mediated changes. Thus, nano size-dependent impacts were evident. Taken together, these experiments suggest that AgNP ingestion, either deliberate or inadvertent, could have negative consequences on our intestinal microbiota.
\end{abstract}

Keywords: Silver nanoparticles; Intestinal microbiota; DGGE; 454-pyrosequencing; Fatty acid methyl esters; Bacterial respiration

\section{Introduction}

The wide-ranging antibacterial, antiviral, and antifungal properties of silver nanoparticles (AgNPs) along with their intrinsic catalytic and optical signatures make these the most popular and commonly used engineered nanoparticles in biomedical applications, cosmetics, clothing, sporting goods, electronics, and of particular significance to this investigation, water purification units, in addition to many other nano-consumer products [1-3]. Currently, 400 products are imbued with engineered AgNPs, comprising more than $51 \%$ of all marketed nano-based consumer products [4]. Given these widespread applications, intentional or accidental release of AgNPs to the environment represents a potential toxicity risk to a wide variety of organisms at many trophic levels [5]. It is known that aquatic and soil microbial communities and their metabolic activities are negatively affected after exposure to AgNPs [6-8]. However, there has been little exploration of the impact of AgNPs on the microbiota associated with living organisms. Of special interest is the effect on the human microbiota considering the range of consumer goods that could lead to exposure through routes such as inhalation, ingestion or direct contact.

The human intestinal microbiota is a diverse microbial ecosystem, with $10^{14}$ intestinal microbes belonging to more than 1000 different species, carrying out metabolic activities that directly influence human health and disease $[9,10]$. The majority $(\sim 99 \%)$ of these bacterial species fall into four major phyla, including the Gram negative Bacteroidetes and the mostly Gram positive Firmicutes (together representing 90\% of the gut bacterial species), as well as Proteobacteria and Actinobacteria $[11,12]$. Reflecting their environment, common human gut genera are either anaerobic (Bifidobacterium, Clostridium, Bacteroides, and Eubacterium) or facultatively anaerobic (Escherichia, Enterococcus, Streptococcus, and Klebsiella) [13]. The taxonomic and phylogenetic composition of these communities can differ among individuals [14]. An intestinal bacterial mixture, "RePOOPulate", is a therapeutic ecosystem comprised of 33 different isolates derived from a healthy human donor [15] that holds promise as a simplified, defined proxy for a healthy gut microbial ecosystem, allowing experimental control and reproducibility [16].

In the fruitfly, Drosophila melanogaster, experimental dietary exposure to AgNPs resulted in the increased midgut abundance of Gram positive genera, chiefly Lactobacillus, at the expense of Gram negative genera such as Sphingdomonas and Rhizobiales, relative to untreated or Ag microparticle-treated controls [17]. This shift in the midgut community was correlated with a reduction in developmental success. To our knowledge, however, the impact of AgNPs on the human intestinal microbiota has not yet been reported. Although it is possible that some human ingestion of AgNPs might occur subsequent to aquatic or soil contamination, we suspect that it is more likely that exposure would be a consequence of the deliberate addition of nanosilver to cosmetics, foods and beverages, in water purifiers, as well as the consumption of silver as an alternative medicine $[1,18,19]$. Here we have modeled such exposure by using "RePOOPulate" as a simplified representation of the human gut microbiota in batch anaerobic fermentation. Polyvinylpyrrolidone (PVP) capped AgNPs were used, as well as ionic silver controls, and across a range of exposure concentrations. We hypothesized that both ionic silver and nanosilver could perturb this microbial community and that there would be a nanosize-dependent impact. We further postulated that if the effect on gut bacteria was significant, then toxicity

*Corresponding author: Pranab Das, Department of Biology, Queen's University, Biosciences Complex, Room 2521, Kingston, ON, K7L 3N6, Canada, Tel: 1-613533-6000; Fax: 1-613-533-6617; E-mail: pd50@queensu.ca

Received September 17, 2014; Accepted October 09, 2014; Published October 18,2014

Citation: Das P, McDonald JAK, Petrof EO, Allen-Vercoe E, Walker VK (2014) Nanosilver-Mediated Change in Human Intestinal Microbiota. J Nanomed Nanotechnol 5: 235. doi: 10.4172/2157-7439.1000235

Copyright: (c) 2014 Das P, et al. This is an open-access article distributed under the terms of the Creative Commons Attribution License, which permits unrestricted use, distribution, and reproduction in any medium, provided the original author and source are credited. 
could be monitored by a suite of assays including respiration, fatty acid methyl esters (FAME), denaturing gradient gel electrophoresis (DGGE) fingerprinting, and DNA sequence analysis. Such analyses could then suggest that deliberate or inadvertent silver exposure, including AgNPs, could result in changes to the gut microbiota, with unknown impacts on overall human health

\section{Materials and Methods}

\section{Preparation of the human microbiota and culture medium}

A synthetic stool mixture of 33 different isolates was prepared by extensive culture as described in detail by Petrof et al. [15]. Briefly, isolates were cultured on fastidious anaerobe agar under anaerobic conditions $\left(90 \% \mathrm{~N}_{2}, 5 \% \mathrm{CO}_{2}, 5 \% \mathrm{H}_{2}\right)$ and subsequently formulated into a predetermined ratio (in $100 \mathrm{~mL} 0.9 \%$ normal saline) to $\sim 3.5 \times 10^{9}$ colony-forming units $/ \mathrm{mL}$. This microbial ecosystem therapeutic bacterial suspension was placed in an air-tight double-sealed container at $4^{\circ} \mathrm{C}$ and used within $2-3 \mathrm{~h}$ of preparation. Autoclaved medium for batch culture experiments was prepared based on a recipe designed for continuous culture of the human distal gut ecosystem [20]. The cultured synthetic stool mixture is hereafter designated as microbial ecosystem therapeutic-1 (MET-1).

\section{Characterization of silver and control materials}

An aqueous suspension of PVP-capped AgNPs was purchased from nanoComposix (San Diego, CA; DAG1964). The manufacturer reported particle sizes of $10.6 \pm 1.7 \mathrm{~nm}$ diameter and a nominal stock concentration of $1.0 \mathrm{mg} / \mathrm{mL}$. Our characterization of the AgNPs was accomplished through dynamic light scattering (DLS), zeta potential and number-based size distributions, respectively, using a Malvern Zetasizer (Malvern Instruments Ltd., UK). Stock suspensions of AgNPs were further size verified by transmission electron microscopy (TEM; Hitachi H-7000, Japan). Particle suspensions of AgNPs in the culture medium at the final concentrations of 25,100 and $200 \mathrm{mg} / \mathrm{L}$ were also confirmed for size using TEM. The actual ${ }^{107} \mathrm{Ag}$ concentration of the stock AgNP suspension was measured by inductively coupled plasmamass spectrometry (ICP-MS; XSeries II; Thermo Scientific, Germany), as described [2], using indium $\left({ }^{115} \mathrm{In}\right)$ as the internal standard. The ICPMS instrument was optimized by Plasmalab software using a $5 \mu \mathrm{g} / \mathrm{L}$ tuning solution of In and Ag. The procedural limit of detection for ${ }^{107} \mathrm{Ag}$ was $0.01 \mu \mathrm{g} / \mathrm{L}$. An aqueous suspension of PVP $(100 \mu \mathrm{g} / \mathrm{mL})$ purchased from nanoComposix (San Diego, CA; DAC1446) was also used as a control for the capping agent.

To prepare the ionic silver controls, the $\mathrm{AgCl}$ suspension procedure was based on Choi et al. [21] with several modifications. Briefly, crystalline $\mathrm{AgNO}_{3}$ and $\mathrm{NaCl}$ (both Fisher Scientific) solutions of 140 $\mathrm{mM}$ and $280 \mathrm{mM}$, respectively, were made separately by dissolving in MilliQ-water. Aliquots of $1000 \mathrm{mg} / \mathrm{L}$ silver chloride $(\mathrm{AgCl})$ suspension were then freshly prepared by vortexing $(\sim 1000 \mathrm{rpm})$ the $\mathrm{AgNO}_{3}$ and $\mathrm{NaCl}$ solutions $(1 \mathrm{~mL}$ each) with MilliQ-water $(18 \mathrm{~mL})$. The $\mathrm{NaCl}$ concentration was twice that of $\mathrm{AgNO}_{3}$ in order to ensure that $\mathrm{AgCl}$ was completely complexed with no residual silver ion $\left(\mathrm{Ag}^{+}\right)$present in the colloidal suspension. The final concentration of $\mathrm{AgCl}$ was determined by confirmatory ICP-MS, as described [6].

\section{Experimental exposures}

For each treatment, MET-1 inocula were added to sterile growth medium (1:10 dilution) in sterile glass serum bottles $(50 \mathrm{~mL}$; Sigma Aldrich), to a final volume of $5 \mathrm{~mL}$, under anaerobic conditions. Triplicate cultures of each of four treatment groups consisted of 25, 100 and $200 \mathrm{mg} / \mathrm{L}$ of AgNPs or AgCl, 2.5, 10 and $20 \mathrm{mg} / \mathrm{L}$ PVP suspensions, as well as control cultures, which were not amended with either Ag or PVP. Since, to our knowledge, there has been no study of AgNPs and human intestinal microbiota; the AgNP concentrations selected were based on other studies concerned with the effects of AgNP on anaerobic pathogenic bacteria [22-24]. We further reasoned that our experiments were of a short-term duration and not chronic exposures, and thus we wanted to include the highest concentrations to model possible in vivo bioaccumulation. Finally, nanosilver water purification units likely leach nanoparticles, based on the wide estimates of release (1-45\%) from different fiber types containing $\sim 0.02-21 \mathrm{mg} / \mathrm{g}$ silver [25]. The PVP doses were chosen as the corresponding concentrations for the amounts used to cap the AgNPs. The inoculated serum bottles were placed at $37^{\circ} \mathrm{C}$ in a Ruskin anaerobic chamber (The Baker Co., Sanford, USA) containing a suitable atmosphere $\left(90 \% \mathrm{~N}_{2}, 5 \% \mathrm{CO}_{2}, 5 \% \mathrm{H}_{2}\right)$. After adding AgNPs, $\mathrm{AgCl}$, or PVP to the stated concentrations, the serum bottles were closed with $20 \mathrm{~mm}$ sterilized rubber stoppers and secured using Wheaton ${ }^{\mathrm{TM}}$ aluminum seals (both Fisher Scientific).The sealed serum bottles were then transferred to a dark room in a sealed vessel containing a Gaspak ${ }^{\mathrm{TM}} \mathrm{EZ}$ anaerobic gas pouch (Fisher Scientific). They were incubated for $48 \mathrm{~h}$ at $37^{\circ} \mathrm{C}$ while being shaken $(\sim 10 \mathrm{rpm})$ on a Bigbill Thermolyne shaker (Dubuque, USA).

\section{Culture head space gas analysis}

Gas generated by MET-1 under experimental conditions was sampled in situ using Luer-Lok ${ }^{\mathrm{TM}}$ Tip BD syringes $(10 \mathrm{~mL})$ with $22 \mathrm{G} 1$ Precision Glide needles (both Becton Dickinson \& Co, NJ) after 24 and $48 \mathrm{~h}$ incubation, and immediately injected using a split-less mode into an Agilent Technologies 7890B Gas Chromatograph (GC; Palo Alto, California, USA), equipped with a stainless steel column (50 $\mathrm{m} \times 0.53$ $\mathrm{mm}$ internal diameter, $10 \mu \mathrm{m}$ film thickness) and packed with Agilent J\&W PoraBOND Q (Palo Alto, California, USA). The operational conditions were as follows: helium $(\mathrm{He})$ gas carrier at $15 \mathrm{~mL} / \mathrm{min}$, thermal conductivity detector (TCD) at $250^{\circ} \mathrm{C}$ and oven temperature at $32^{\circ} \mathrm{C}$ for $5 \mathrm{~min}$. Of the three major universal components of human flatus $\left(\mathrm{CO}_{2}, \mathrm{H}_{2}\right.$ and $\mathrm{N}_{2}$; [24]) only two were assayed with our equipment; peak areas of $\mathrm{CO}_{2}$ and $\mathrm{N}_{2}$ were quantified with Agilent Technologies ChemStation Integration Software (Palo Alto, California, USA). It should be noted that because MET-1 contains no Archaea, methane production was not measured.

\section{Fatty acid methyl ester (FAME) analysis}

Triplicate subsamples $(1 \mathrm{~mL}$ each) for each treatment group were centrifuged at $2000 \mathrm{xg}$ for $10 \mathrm{~min}$ and the pellets stored at $-80^{\circ} \mathrm{C}$ until analysis (Keystone Labs; Edmonton, Canada). Phospholipid fatty acids were extracted from the pellets using the MIDI Sherlock Microbial Identification System (Microbial ID Inc., Newark, DE, USA) as stated elsewhere [26]. Briefly, each pellet was saponified with $1 \mathrm{~mL}$ of $\mathrm{NaOH}$ in aqueous methanol $(15 \% \mathrm{w} / \mathrm{v})$ at $100^{\circ} \mathrm{C}$ for $30 \mathrm{~min}$, followed by methylation with $2 \mathrm{~mL} \mathrm{HCl}$ in aqueous methanol $(54 \% \mathrm{w} / \mathrm{v})$ at $80^{\circ} \mathrm{C}$ for $10 \mathrm{~min}$. Subsequent extraction used $1.25 \mathrm{~mL}$ of hexane/methyl-tertbutyl ether $(50 \% \mathrm{v} / \mathrm{v})$ for $10 \mathrm{~min}$. After discarding the aqueous phase, the samples were washed for $5 \mathrm{~min}(3 \mathrm{~mL}$ of $0.3 \mathrm{M} \mathrm{NaOH}$ containing a few drops of saturated $\mathrm{NaCl}$ ). The organic phase was analyzed with a GC equipped with an Ultra 2 column containing a flame ionization detector (Agilent). The resulting chromatographic peak areas were converted to the percentage of the total fatty acids (mol \%) for each sample. The more abundant fatty acids $(>0.15 \% \mathrm{~mol} \%)$ were subjected to further analysis. In addition, all fatty acids were sub-grouped into Gram positive, Gram negative and saturated categories, as outlined elsewhere [27]. 


\section{DNA isolation}

At the conclusion of the $48 \mathrm{~h}$ incubation period, samples $(200 \mathrm{uL})$ were removed from each serum bottle for genomic DNA extraction using a QIAamp DNA stool mini kit (Qiagen Sciences, LLC, MD, USA), as described in the manufacturer's protocol. The concentration and purity of the DNA was assessed by spectrophotometric analysis (Ultrospec $^{\mathrm{TM}} 1100$ pro UV/visible Spectrophotometer; Amersham Biosciences Corp., USA, as well as a NanoDrop-1000; Ver.3.7.1; Thermo Scientific, Wilmington, USA) and by polymerase chain reaction (PCR) followed by agar gel electrophoresis as described below.

\section{PCR-DGGE}

Genomic DNA was used as a template for PCR amplification of 16S ribosomal RNA (rRNA) genes using the forward primer, 341F (5'-CCTACGGGAGGCAGCAG) with an additional 40-nucleotide GC-rich sequence on the 5'-end (GC clamp) and the reverse primer, 518R (5'- ATTACCGCGGCTGCTGG; [27]). PCR was performed with a $50 \mu \mathrm{L}$ reaction mixture containing $4 \mu \mathrm{L}$ of DNA template, 2.5 $\mu \mathrm{L}$ of each primer $(10 \mu \mathrm{M}), 5 \mu \mathrm{L}$ of $10 \mathrm{X}$ ViBuffer A (Vivantis), $1.5 \mu \mathrm{L}$ of $\mathrm{MgCl}_{2}(50 \mathrm{mM}), 0.5 \mu \mathrm{L}$ of dNTP mix (10 mM; Thermo Scientific), $2.5 \mu \mathrm{L}$ of bovine serum albumin $(2 \mathrm{mg} / \mathrm{mL}), 0.4 \mu \mathrm{L}$ of recombinant $\mathrm{Taq}$ DNA polymerase $(10 \mathrm{mM}$; Vivantis) and $31.1 \mu \mathrm{L}$ sterile water using the following protocol: $5 \mathrm{~min}$ at $94^{\circ} \mathrm{C}$ denaturation, followed by a total of 30 cycles $94^{\circ} \mathrm{C}$ for $1 \mathrm{~min}, 64^{\circ} \mathrm{C}$ for $1 \mathrm{~min}, 72^{\circ} \mathrm{C}$ for $3 \mathrm{~min}$, followed with a final extension at $72^{\circ} \mathrm{C}$ for $7 \mathrm{~min}$. Negative controls without the DNA template were always included as controls. PCR amplification was performed in a Veriti 96 Well Thermal Cycler (Applied Biosystems, Burlington, Canada). The presence and integrity of the amplified products were confirmed by electrophoresis in $1 \%$ (wt/vol) agarose gels stained with ethidium bromide and viewed on a UV transilluminator (Alpha Innotech, Santa Clara, USA).

Amplified 16S rRNA gene fragments were subjected to DGGE as described elsewhere [28] using a DGGEK-2401 System (CBC Scientific, Del Mar, USA). Briefly, amplified PCR products $(20 \mu \mathrm{L}$ of each triplicate sample for each treatment groups) were loaded onto $8 \%$ $(\mathrm{w} / \mathrm{v})$ polyacrylamide gels containing a urea gradient ranging from $30 \%$ to $60 \%$.The gels were electrophoresed at $60^{\circ} \mathrm{C}$ in $1 \mathrm{X}$ Tris-acetate-EDTA (TAE) buffer; after the amplified products had entered the gels $(120 \mathrm{~V}$ for $5 \mathrm{~min}$ ), a constant voltage was applied ( $70 \mathrm{~V}$ for $20 \mathrm{~h}$ ). Following electrophoresis, the gels were incubated for $30 \mathrm{~min}$ in $1 \mathrm{X}$ TAE buffer containing ethidium bromide $(0.5 \mathrm{mg} / \mathrm{L})$ and then rinsed with TAE buffer for 10-12 min. Stained gels were visualized with an Alpha Innotech UV transilluminator and photographed using AlphaEase FC software (Version 6.0, Alpha Innotech, Santa Clara, CA, USA). Images of DGGE-generated rRNA profiles were analyzed with Syngene Genetools software (version 4.03.03, Synoptics Ltd.).

\section{Pyrotag sequencing}

The 454 pyrotag sequencing of MET-1 treatment groups followed standard protocols (MR DNA; Shallowater, TX, USA). Briefly, sequence corresponding to the $16 \mathrm{~S}$ rRNA V1 to V3 hypervariable regions ( $\sim 500 \mathrm{bp}$ fragments) from each triplicate DNA sample and each concentration in the four treatment groups was independently PCR amplified with primer pairs consisting of a forward primer, Gray 28F (5'-TTTGATCNTGGCTCAG) and reverse primer, Gray 519r (5'-GTNTTACNGCGGCKGCTG). A single-step PCR using HotStarTaq Plus Master Mix Kit (Qiagen, Valencia, CA) was initiated by incubating template DNA for $3 \mathrm{~min}$ at $94^{\circ} \mathrm{C}$, and was followed by 28 cycles of $94^{\circ} \mathrm{C}$ for $30 \mathrm{~s}, 53^{\circ} \mathrm{C}$ for $40 \mathrm{~s}$, and $72^{\circ} \mathrm{C}$ for $1 \mathrm{~min}$, with a final 5 min extension at $72^{\circ} \mathrm{C}$. Amplified products were purified using Agencourt Ampure beads (Agencourt Bioscience Corporation, Beverly, USA). Amplified DNA ( $500 \mathrm{ng}$ ) was sequenced using the Genome Sequencer FLX System (Roche, Nutley, USA) using titanium reagents as described by the manufacturer's protocols [29]. After removing failed sequence reads, as well as low quality sequence and primers, any nonbacterial reads and chimeras were removed using Black Box Chimera Check (B2C2) software [30,31]. Sequencing reads were subsequently assembled into clusters using a distributed MegaBLAST.NET algorithm [32] after denoising, and compared to the $16 \mathrm{~S}$ sequences of the National Center for Biotechnology Information (NCBI) database library RDP ver 9 [33]. MegaBLAST outputs were further evaluated using .NET and C\# analysis pipeline [29]. The bacterial identity was based on the percentage of the total length of each sequence aligned with the given library database sequence, and in comparison with the known MET-1 sequences. The 454 pyrosequencing distributions in each treatment group were compared as such, or normalized using the corresponding total mol\% fatty acids.

\section{Statistical analysis}

The impact of AgNPs, $\mathrm{AgCl}$ and PVP treatments on gas production, FAME analysis and DNA sequence were evaluated with two-way analysis of variance (ANOVA) using SPSS (SPSS Inc., Chicago, USA). Tukey's HSD for honest significant difference post-hoc test evaluated significance between any two pairs of means. Hierarchical clustering of DGGE band profiles were based on banding patterns, and intensity was done using Pearson correlation coefficient and UPGMA (Unweighted Pair Group Method with Arithmetic Mean) methods. Multidimensional scaling (MDS) of the percentage of the total length of each phylogenetic sequence was used to reduce the bacterial composition dataset and compare multivariate responses to the concentrations of $\mathrm{AgNPs}, \mathrm{AgCl}$ or PVP exposures including the control groups (no Ag or PVP) using SAS Ver 9.4 (SAS Institute, Inc., Cary, USA).

\section{Results}

\section{Nanoparticle characterization}

The number-based hydrodynamic size distribution (Malvern Zetasizer) showed that the average particle size of the AgNP stock suspension was $\sim 10.87 \pm 1.2 \mathrm{~nm}$ (Figure 1A). TEM analysis of this suspension also confirmed the manufacturer's mean particle size data (Figure 1B). The zeta potential of $-25.2 \mathrm{mV}$, again, was reasonably consistent with that of the supplier. Since the insoluble micron-size components of the culture medium precluded an accurate assessment of AgNP hydrodynamic size, TEM was used to show that the AgNPs were well dispersed in the medium in a dose-dependent manner, again with sizes within the range described (Figures 1C, 1D and 1E). Importantly, these TEM images showed that any clusters consisted of single NPs rather than agglomerated clumps.

\section{Bacterial gas production in the cultures}

MET- 1 control cultures generated $\mathrm{CO}_{2}, \mathrm{~N}_{2}$, and unidentified gas in the head space, with the total gas representing about double the culture volume after $48 \mathrm{~h}$ (Figure 2). All PVP-treatment groups showed $\mathrm{CO}_{2}$ and $\mathrm{N}_{2}$ gas production as well as total gas volumes that were the same as controls, indicating that the molecule used as a capping agent for the AgNPs had no apparent influence on bacterial respiration. Notably however, total gas volume was significantly reduced $(\mathrm{p}<0.05)$ in all silver treatment groups, except at the lowest AgNP concentration. When MET-1 cultures included 100 and $200 \mathrm{mg} / \mathrm{L}$ AgNPs (Figure 2), the total head space gas was significantly reduced ( $\mathrm{p}<0.05$ ) by $\sim 20 \%$ and with 
Citation: Das P, McDonald JAK, Petrof EO, Allen-Vercoe E, Walker VK (2014) Nanosilver-Mediated Change in Human Intestinal Microbiota. J Nanomed Nanotechnol 5: 235. doi: 10.4172/2157-7439.1000235
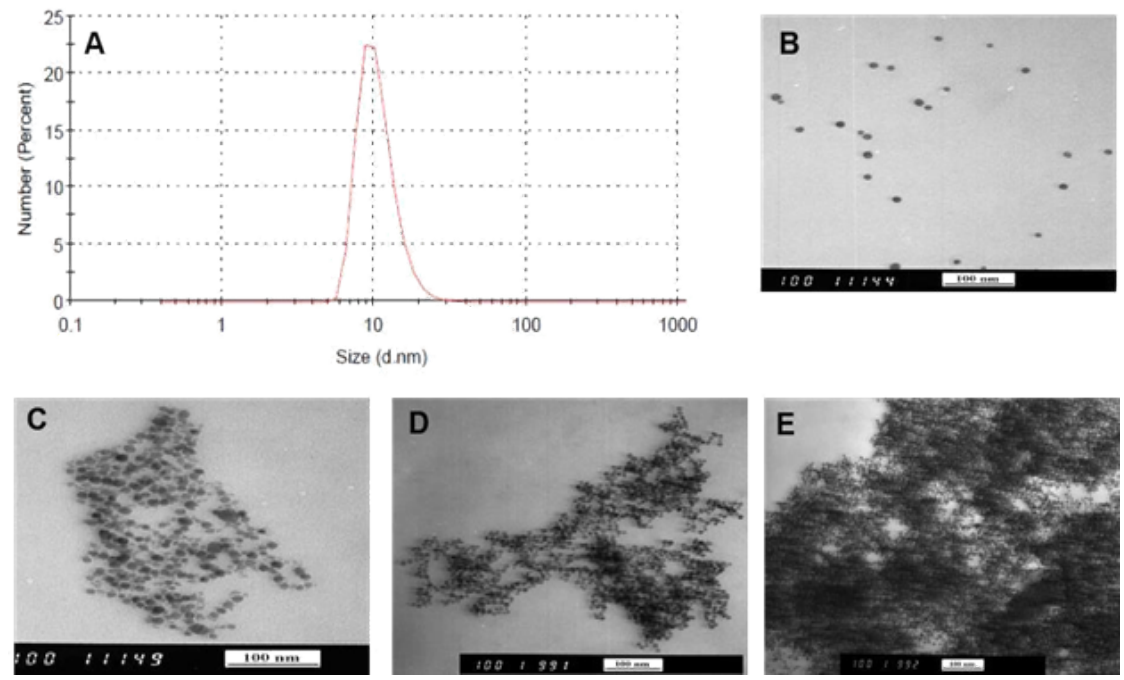

Figure 1: Characterization of silver nanoparticles (AgNPs): A) dynamic light scattering (DLS) number-based size distribution vs. an X-axis logarithmic scale (diameter in $\mathrm{nm}$; d.nm); B) transmission electron micrographs (TEM) image of the AgNP stock suspension; and also TEM images of AgNPs in the bacterial culture media at C) 25, D) 100 \& E) $200 \mathrm{mg} / \mathrm{L}$ concentrations.

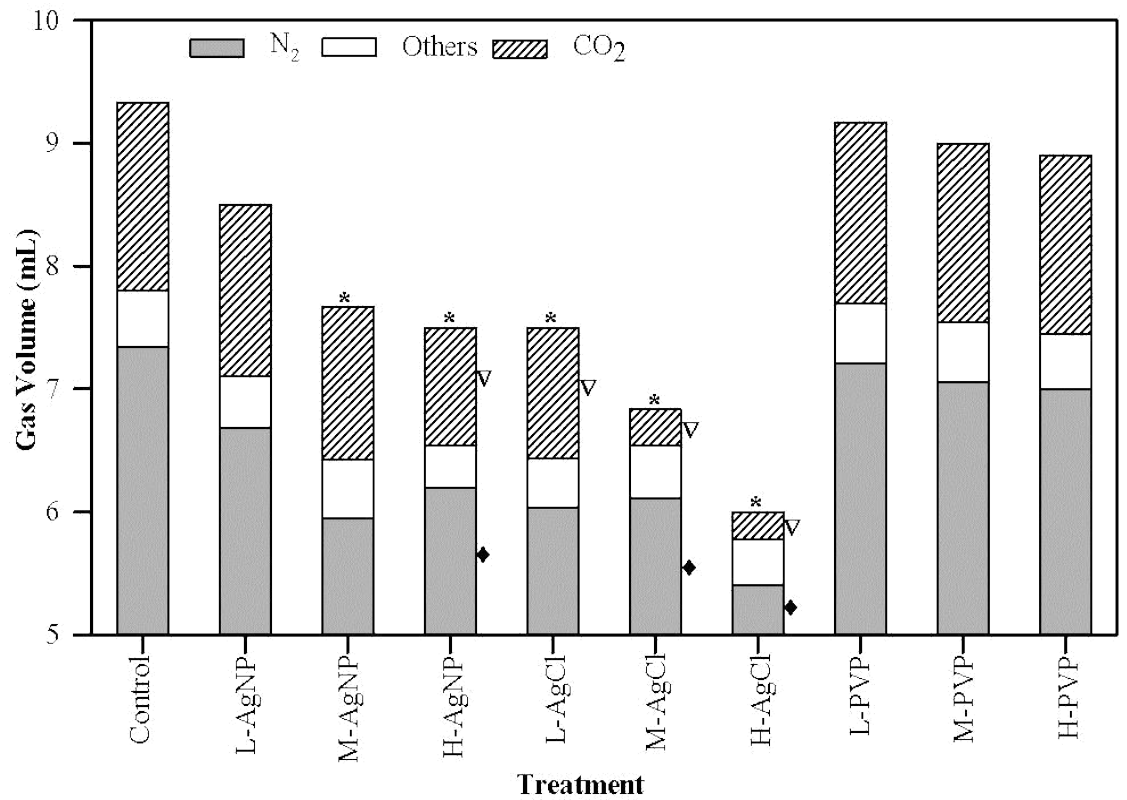

Figure 2: Carbon dioxide $\left(\mathrm{CO}_{2}\right)$ and nitrogen $\left(\mathrm{N}_{2}\right)$ gas recovered from the total gas generated by the human therapeutic intestinal bacterial communities after 48 $\mathrm{h}$ exposure to silver nanoparticles (AgNPs), silver chloride (AgCl), polyvinylpyrrolidone (PVP) and control (with no Ag or PVP), where $\mathrm{L}$, M and $\mathrm{H}$ before AgNP and $\mathrm{AgCl}$ represent 25,100 and $200 \mathrm{mg} / \mathrm{L}$ and L, M, H before PVP represent $2.5,10$ and $20 \mathrm{mg} / \mathrm{L}$ concentrations, respectively, in the X-axis. The symbols '*', ' $\nabla$ ' and ' $\$$ ' show significance differences $(p<0.05)$ in the total gas volume, $\mathrm{CO}_{2}$ and $\mathrm{N}_{2}$, respectively, compared to the control.

$\mathrm{CO}_{2}$ reduced to $\sim 22 \%$ of control values. Similarly, total gas volumes were significantly reduced by $\sim 35 \%(p<0.05)$ in the $25-200 \mathrm{mg} / \mathrm{L} \mathrm{AgCl}$ treatment groups. As with the AgNP amendments, this reduction was correlated with a lower production of $\mathrm{CO}_{2}$, which was significantly reduced $(p<0.05)$ by $20-78 \%$ at increasing $\mathrm{AgCl}$ concentrations. The reduction in bacterial respiration was so prominent in the $200 \mathrm{mg} / \mathrm{L}$ $\mathrm{AgNP}$ and $\mathrm{AgCl}$ treatment groups, as well as in the $100 \mathrm{mg} / \mathrm{L} \mathrm{AgCl}$ cultures, that the proportion of $\mathrm{N} 2$ head space gas was significantly increased $(p<0.05)$ relative to controls.

\section{Fatty acid profiles after culture}

A total of 31 different phospholipid fatty acid peaks were seen in chromatographs of MET-1 cultures. Of these, 23 dominant (> $0.15 \mathrm{~mol} \%$ ) fatty acids were selected for comparative analysis and characterized into Gram negative, Gram positive, saturated and unnamed groups (Figure 3). Fatty acid signatures in the PVP treatment groups were identical to controls (Figure S1). In contrast, after the silver treatments, overall fatty acid profiles were strikingly different. For example, saturated fatty acids were significantly affected $(p<0.05)$ 
Citation: Das P, McDonald JAK, Petrof EO, Allen-Vercoe E, Walker VK (2014) Nanosilver-Mediated Change in Human Intestinal Microbiota. J Nanomed Nanotechnol 5: 235. doi: 10.4172/2157-7439.1000235

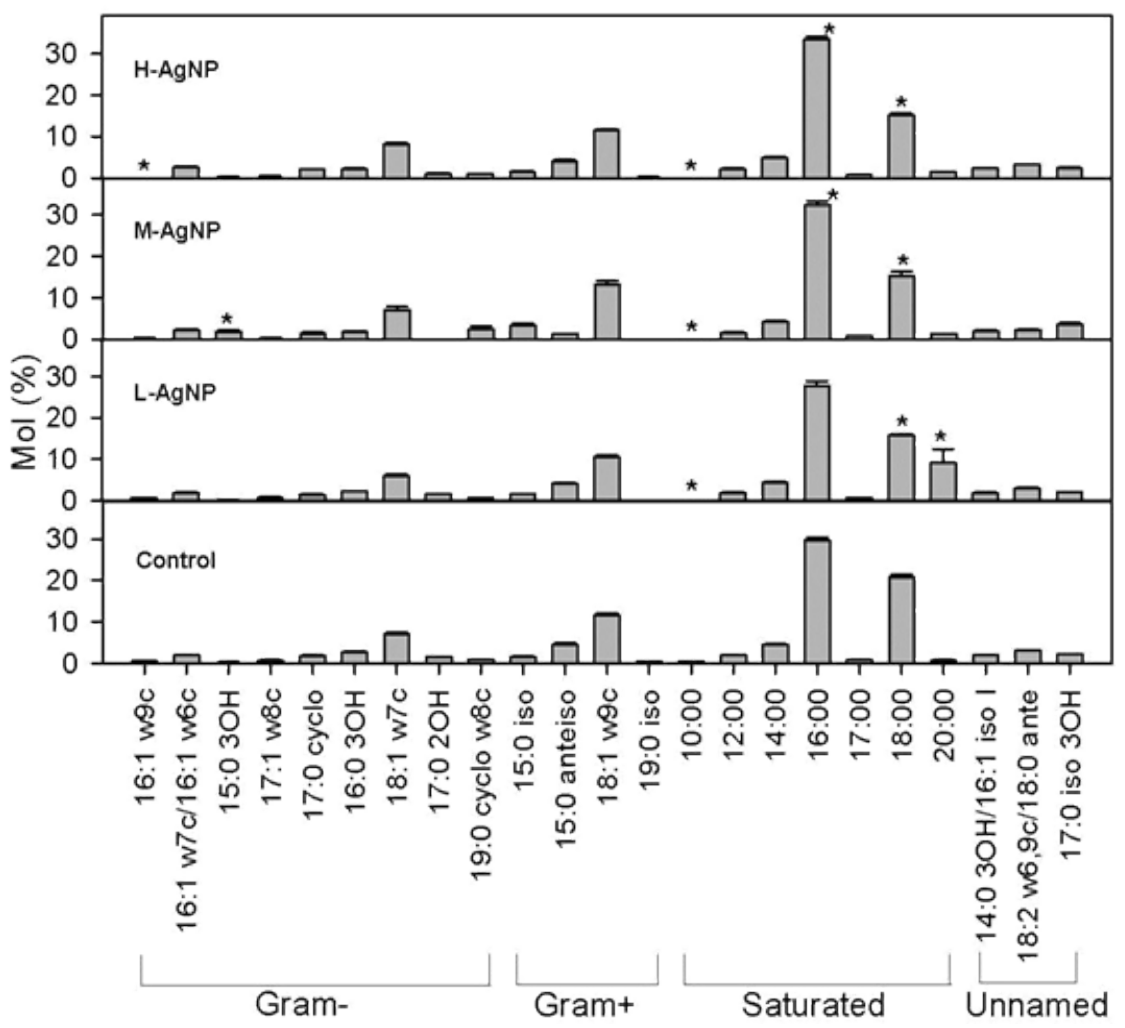

Figure 3: Relative amounts (mol\%) of extracted fatty acids in control (no AgNPs) and AgNPs treatments at 25 (L-AgNP), 100 (M-AgNP) and 200 (H-AgNP) mg/L concentrations, respectively. The bars represent the means of three independent fatty acid assessments and the standard errors. The fatty acids are grouped according to their most-frequently associated categories. Unknown lipid indicators were grouped as 'Unnamed'. The symbol '*' show significant differences $(p<0.05)$ compared to the control.

by AgNP exposure compared to the controls: capric (10:0) and stearic (18:0) fatty acids were reduced by $80-96 \%$ and $20-26 \%$, respectively (AgNPs at 25-200 $\mathrm{mg} / \mathrm{L}$ ), and the most abundant saturated palmitic fatty acid (16:0) increased by $26-32 \%$ (AgNPs at $200 \mathrm{mg} / \mathrm{L}$ ). $\mathrm{AgCl}$ treatments had similar effects: 10:0 and 18:0 fatty acids were decreased by $65-90 \%$ and $30-35 \%$, respectively $(\mathrm{AgCl}$ at $25-200 \mathrm{mg} / \mathrm{L}$ ), and $16: 0$ fatty acids increased by $13-28 \%$, compared to controls (Figure S2). Even at the lowest AgNP concentration there was an impact, including fatty acid 20:00 (arachidic acid), which increased to $9 \mathrm{~mol} \%$ from $1.1 \mathrm{~mol} \%$ in controls (Figure 3). There were no significant differences noted in fatty acid signatures normally associated with Gram positive or Gram negative bacteria (except 16:1 $\omega 9 \mathrm{C}$ and 15:03OH in the 100 and $200 \mathrm{mg} / \mathrm{L}$ AgNP concentrations, respectively). In contrast, fatty acids in the AgCltreatment groups showed more impact with changes in Gram positive signature fatty acids (e.g. 15:0 anteiso and 18:1 $\omega 9 \mathrm{C}$ unsaturated fatty acids were increased by $20-45 \%$ ), and most Gram negative signatures were reduced by $15-100 \%$ (Figure S2). Taken together, regardless of any increase or decreases, the total fatty acids in MET- 1 treatment groups were positively correlated $(r=0.682 ; p<0.01)$ with changes in the total gas volume seen after $48 \mathrm{~h}$ exposure to silver, either as $\mathrm{AgNP}$ or $\mathrm{AgCl}$, indicating that these two assessments were concordantly indicative of $\mathrm{AgNP}$ and AgCl-mediated toxicity.

\section{The effect of silver treatment on PCR-DGGE profiles}

MET-1 DNA analysis by DGGE showed differences in banding patterns and intensities in the silver and control treatment groups. In this regard, the PVP-treatment groups were identical to non-amended controls (Figure S3). After AgNP exposure, some banding groups were either more or less intense compared to controls (band group a1; Figure 4), some showed little change (band group a3 and a5), and at the highest AgNP concentration, one (band group a2) completely disappeared. Still another became apparent (band group a6) that had previously been below the detection point. Cluster analysis showed that the DGGE banding profiles of the MET-1 AgNP-treatments grouped separately from control cultures; they differed $26 \%-36 \%$ at increasing AgNP concentrations. DGGE results also differed in the AgCl-treatment groups and generally showed somewhat greater divergence from control patterns than even those seen after AgNP amendment. For example, $\mathrm{AgCl}$ MET-1 patterns differed up to $42 \%$ of controls (Figure S4).

\section{The effect of silver treatment on the phylogenetic composition}

Because of distinct differences seen in respiration, fatty acids and DGGE patterns, DNA from each of the different triplicate MET-1 treatment groups was sequenced. Due to possible sequencing errors, only those reads that had a relative abundance of $\geq 1 \%$ were used in the analysis; minor contributors to MET-1 were placed together as a single 'Others' group for each treatment. It should be noted that although the contribution of the 'Others' category varied somewhat between exposure groups, there were no significant differences between them, including in the controls (Figure 5). As well, regardless of the PVP concentration, there were no significant differences in MET-1 phylogenetic compositions and the unamended controls (Figure S5). MDS analysis after PVP treatments showed a patchy distribution that was scattered among treatments including control (Figure 6; right 
Citation: Das P, McDonald JAK, Petrof EO, Allen-Vercoe E, Walker VK (2014) Nanosilver-Mediated Change in Human Intestinal Microbiota. J Nanomed Nanotechnol 5: 235. doi: 10.4172/2157-7439.1000235

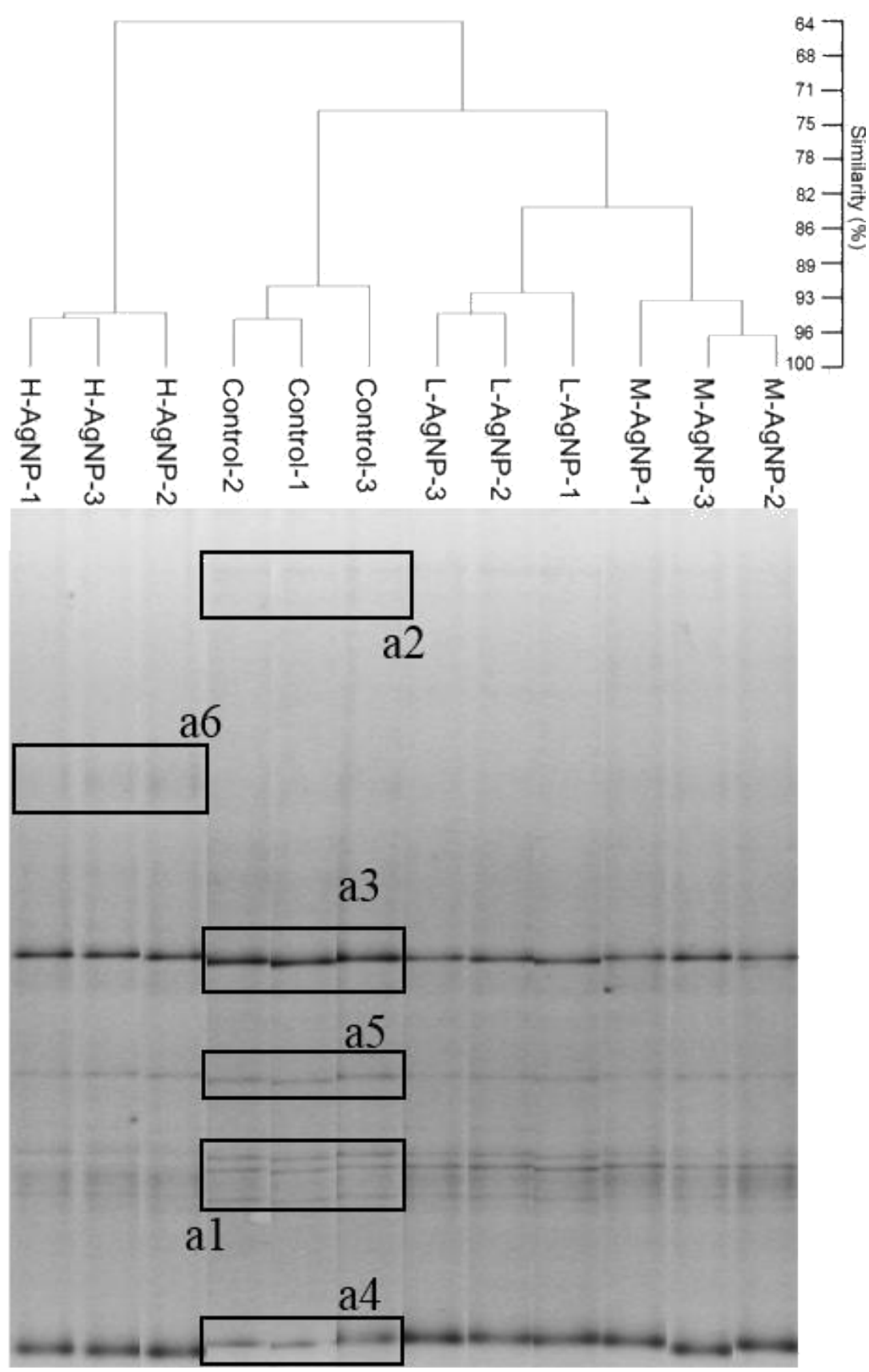

Figure 4: UPGMA clustering analysis using DGGE band patterns after exposure to silver nanoparticles (AgNPs) and control (with no AgNP), where L, M and $H$ before AgNP represents 25,100 and $200 \mathrm{mg} / \mathrm{L}$ concentrations, respectively. Numbers at the end of the descriptors $(1,2,3)$ indicate the replicate samples. Band groups a1-a6 represent highlighted differences in banding pattern between controls and AgNP treatments.

panel).

After AgNP treatments, the phylogenetic assemblages were significantly impacted $(p<0.01)$ relative to controls (Figure 5$)$, and as suggested by the DGGE results. The MET-1 ecosystem is dominated by a strict anaerobe, Bacteroides ovatus ( 35\%), but after AgNP amendment, its abundance was reduced by $\sim 57 \%$, while the Gram negative, Raoultella sp. ( 23\%) and Escherichia coli (6\%) were increased by $28-46 \%$ and $50-80 \%$, respectively, at the various AgNP concentrations (Figure 5). Other anaerobes such as Roseburia faecalis ( 5.5\%), Eubacterium rectale
( $5 \%)$, Roseburia intestinalis (4\%) and Ruminococcus torques( 2\%) were also significantly decreased $(p<0.01)$ by $30-95 \%, 45-97 \%, 64-81 \%$ and 44-70\%, respectively, in the AgNP exposure groups relative to controls (Figure 5). MDS analysis demonstrated that AgNP treatment groups were distinct from controls. Although MET-1 shifted in response to AgNP amendment at all of the tested concentrations, the two lower concentration treatments grouped together and were separated from the highest AgNP concentration (Figure 6; left panel). Notably, the impact of AgNPs on the human intestinal bacteria was apparent whether or 
Citation: Das P, McDonald JAK, Petrof EO, Allen-Vercoe E, Walker VK (2014) Nanosilver-Mediated Change in Human Intestinal Microbiota. J Nanomed Nanotechnol 5: 235. doi: 10.4172/2157-7439.1000235

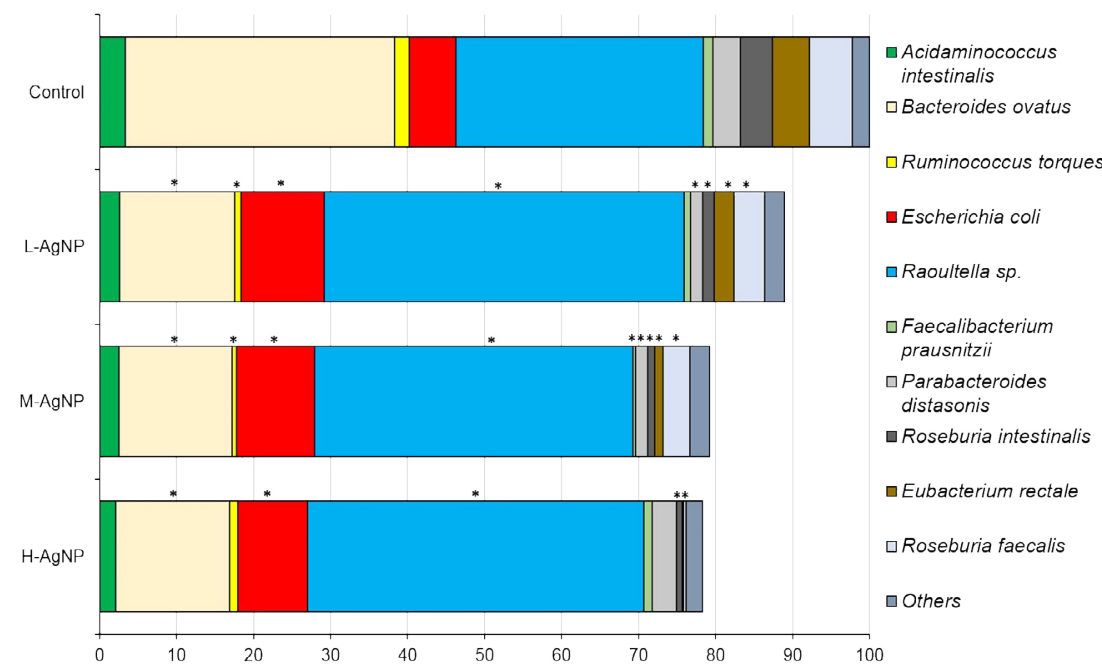

Figure 5: Human intestinal bacterial phylogenetic composition normalized to their total fatty acid responses at the species level after $48 \mathrm{~h}$ exposure to 0 (Control), 25 (L-AgNP), 100 (M-AgNP), and 200 (H-AgNP) mg/L silver nanoparticles (AgNP), respectively. Means of the triplicate samples in each treatment with $>1 \%$ relative abundance were used for sequence identity, whereas groupings of less abundant species shown as 'Others'. Asterisk (*) symbol represents significant differences $(p<0.01)$ at species level compared to the control. Non-normalized graphs are shown in Figure S6.
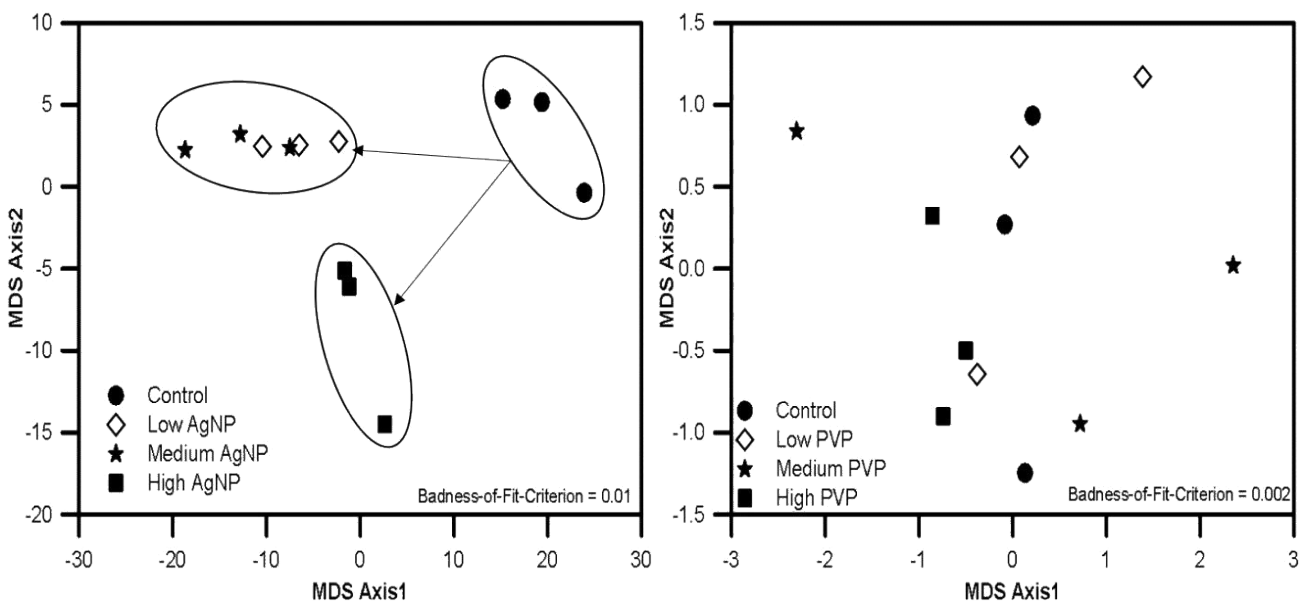

Figure 6: Multidimensional scaling (MDS) plots of human intestinal bacterial community composition based on pyrosequencing after $48 \mathrm{~h}$ exposure to Left Panel: 0 (Control), 25 (Low), 100 (Medium) and 200 (High) mg/L silver nanoparticles (AgNP), and Right Panel: 0 (Control), 2.5 (Low), 10 (Medium) and 20 (High) mg/L polyvinylpyrrolidone (PVP) concentrations, respectively. Arrows indicate the distance of the AgNP- treatment groups from the control with no Ag.

not the sequence distribution was normalized for total abundance or remained as a distribution percentage (Figure 5; Figure S6).

Similar, but depending on the target bacteria sometimes more disruptive to MET-1 than the AgNP treatments, was exposure to AgCl. For example, the abundance of $B$. ovatus and $R$. torques was significantly reduced $(p<0.001)$ by $84 \%$ and $80 \%$, respectively, at $25 \mathrm{mg} / \mathrm{L} \mathrm{AgCl}$, but there was no significant impact on these two bacteria at the higher $\mathrm{AgCl}$ concentrations (Figure S5). The abundance of Raoultella sp. and E. coli were significantly increased $(p<0.01)$ at the lowest $\mathrm{AgCl}$ concentrations, as they were after the AgNP treatments. However, they significantly decreased ( $p<0.01 ; 64 \%$ and $65 \%$, respectively) in the highest $\mathrm{AgCl}$ concentrations (Figure S5). Similar to the effects of AgNPs, $R$. faecalis, E. rectale, $R$. intestinalis and Acidaminococcus intestinalis (2.3\% of controls) were significantly reduced by $91-95 \%$,
82-96\%, 76-92\% and 84-92\%, respectively, after $\mathrm{AgCl}$ treatments. Distinct from AgNP effects, however, there was also a significant reduction $(p<0.05)$ in the phylogenetic composition of the 'Others' group in $\mathrm{AgCl}$ treatments compared to the non-exposed group. Indeed, four anaerobes in the 'Others' group including Collinsella aerofaciens ( $\sim 0.16 \%)$, Dorea longicatena ( 0.2\%), Eubacterium desmolans ( $0.13 \%)$ and Ruminococcus obeum ( 0.1\%) appeared to be eliminated after $\mathrm{AgCl}$ exposure (Figure S6). In all, MDS analysis revealed a clear distinction in phylogenetic composition between control and $\mathrm{AgCl}$ treatments, while communities treated with the lowest $\mathrm{AgCl}$ concentration separated from that of the higher $\mathrm{AgCl}$ treatments and control, respectively (Figure S7). Significantly, MDS analysis showed differences between $\mathrm{AgCl}$ and $\mathrm{AgNP}$ treatments. This again underscores the distinction between the two forms of silver amendment and suggests some distinct nano-specific-effects. 


\section{Discussion}

With the increased availability of nano-consumer products, including cosmetics, foods and beverage supplements, as well as water purifiers and the use of alternative medicines $[1,18,19]$, there is little doubt that we will ingest some nanosilver. Will this exposure have an impact on our intestinal microbiota? The results of these experiments suggest that at a certain threshold concentration, it will.

Since to our knowledge, this question had not been previously addressed, there were few studies on which to model our experiments. As a result, four different assays, representing three distinct physiological and cellular responses were used including respiration, fatty acids and DNA analysis. Initially, we doubted that the monitoring of respiration gases over a period of only $48 \mathrm{~h}$ would be sensitive enough to detect any toxicological impact. After all, no significant effect on gas production was observed in anaerobic microbial communities after exposure to $\mathrm{C}_{60}$ fullerenes over an exposure period of a few months [34]. However, total gas production was significantly reduced in almost every experimental treatment (100-200 mg/L AgNP or 25$200 \mathrm{mg} / \mathrm{L} \mathrm{AgCl}$ ) compared to controls (Figure 2). The lower volumes appeared to be correlated with a reduction in the generation of $\mathrm{CO}_{2}$, a fermentation product of carbohydrate metabolism. This suggests that silver amendment could substantially reduce the metabolic activity of these cultures. Interestingly, at tested $\mathrm{AgCl}$ concentrations $\geq 100$ $\mathrm{mg} / \mathrm{L}$, the proportion of the measured gases remained the same as the starting gas concentrations. Thus, notwithstanding the depressed level of fermentation to $\mathrm{CO}_{2}$, there must have been some anaerobic respiration yielding $\mathrm{N}_{2}$ to replace the gas withdrawn by sampling. E.coli, which can reduce nitrite and consequently have been implicated in the pathology of inflammatory bowel disease [35], increased in abundance (50-80\%) after AgNP treatments (25-200 mg/L; Figure 5), and these as well as other bacteria (e.g. Raoultella sp.) may have contributed to the maintenance of $\mathrm{N}_{2}$ at a high level. After $\mathrm{AgCl}$ amendments, there was an increase $(56-191 \%)$ in the relative proportion of Parabacteroides distasonis (Figure S5; S8), an isolate which would also contribute to $\mathrm{N}_{2}$ generation since it can use nitrate as a terminal electron acceptor for anaerobic respiration [36].

The silver-mediated reduction in gas production associated with lower metabolic activity, likely reflected a reduction in cell division [37], and this was positively correlated $(r=0.68)$ with the total fatty acids recovered. Together, the FAME analysis and the respiration assay provided an estimate of the bacterial mass at the conclusion of the incubation period. Fatty acid profiles differed considerably between silver treatment groups and the controls.

It has been suggested that the thicker peptidoglycan layer of Gram positive species may act as a barrier to NPs and make these cells less susceptible to AgNP toxicity $[17,38]$ and here the abundance of oleic acid $(18: 1 \omega 9 \mathrm{c})$, a Gram positive biomarker, was not significantly perturbed after AgNP treatments. However, it must be noted that such 'signature' assignments can only be applied with caution since stress can induce changes in membrane lipids [39]. Indeed, we included FAME analysis to test this premise; had fatty acid signatures deviated in the absence of a community shift, future investigators would know to avoid FAME analysis for AgNP toxicity tests. Gram negative bacteria with their thinner cell membranes could be more vulnerable to an AgNP-mediated increase in unsaturated fatty acids resulting in a disruption of membrane permeability and ultimately resulting in cell death $[40,41]$. In this regard, the most predominant Gram negative, $B$. ovatus, was reduced $\sim 57 \%$ after AgNP treatment, compared to controls.
$\mathrm{AgCl}$ treatments also resulted in changes to the pattern of fatty acid signatures with an increase in saturated fatty acids and a concomitant decrease in unsaturated Gram negative fatty acid biomarkers. However, not enough is known about the interaction of silver ions with consortia to make definite conclusions. P. distasonis, a Gram negative which represented $\sim 4 \%$ of the sequences in controls, more than doubled in relative abundance at high $\mathrm{AgCl}$ concentrations. This increase was also reflected in the significant elevation of major fatty acids in this species [42] including 17:0 iso $3 \mathrm{OH}$, an 'unnamed' fatty acid, as well as $18: 1$ $\omega 9 \mathrm{c}$, ironically a 'signature' Gram positive marker. Thus, although stress can undoubtedly change fatty acid profiles in individual bacteria, our results indicate that fatty acid profiles coupled with respiration assessments may well find applicability as an efficient screening method for the impact of NPs on intestinal bacterial communities.

Since together these analyses indicated that there were changes in the MET- 1 community structure, even at the lowest concentrations in each treatment group, it was important to determine if such differences were reflected in a shift in species abundance. Initially, we examined this using PCR-DGGE. It was not possible to identify individual species by the amplified rRNA gene fragments since most of the genomes in MET1 have similar overall GC content of around 50\%, however, we noted variations in band intensities in both the $\mathrm{AgNP}$ and $\mathrm{AgCl}$ treatment groups (Figure 4; Figure S4). UPGMA clustering analysis with these DGGE banding patterns demonstrated that the AgNP-treatment groups were $\sim 36 \%$ different that those in control, with the lower levels (25 and $100 \mathrm{mg} / \mathrm{L}$ AgNPs) clustering closer together and distinct from the $200 \mathrm{mg} / \mathrm{L}$ AgNP amendment group. UPGMA clustering analysis with the $\mathrm{AgCl}$ amendment experiments showed a dose effect with the lower $\mathrm{AgCl}$ concentration more similar to controls than at higher concentrations. We assume that these observed differences likely reflect the more active division of bacteria that were less sensitive to either $\mathrm{AgNP}$ or $\mathrm{AgCl}$. It is well known that resistance to silver can be due to the selective growth of dominant or Ag-tolerant species with resistance genes [43], and such differential sensitivities to this metal have been seen previously in soil studies $[8,44]$. What is notable, however, is that the impact of silver, whether in the NP form or the ionic form, appeared to be distinct, resulting in dissimilar banding patterns.

AgNP-mediated changes in MET-1 as evidenced by PCR-DGGE or pyrosequencing, and subsequently assessed by clustering and MDS analysis, respectively, were concordant (Figures 4-6). This suggests that changes in the community structure for large numbers of samples, such as dose-dependent screens on the impact of various NPs on the human gut ecosystem could be achieved using gel analysis. Pyrosequencing, however, indicated specific targeted members of the MET- 1 community. $\mathrm{AgCl}$ treatment was toxic to some MET-1 members and shifted the community more radically than the AgNP treatments (Figures 5 and S5). Nonetheless, even at the lowest concentrations of AgNP, there were significant reductions in the relative abundance of individual bacteria, either when normalized to total gases (not shown), total fatty acids (Figure 5), or when based on overall distributions (Figure S6). Although we did not use a complex fecal microbial consortium, but rather a defined, simplified, and highly reproducible human distal gut ecosystem [15], many of these microbes demonstrated differences in their susceptibility to AgNPs. For some of these microbes, there could be a negative effect of AgNPs on the overall health of the host. For example, the abundance of $B$. ovatus, which provides energy in the form of short-chain fatty acids and sugars that mediate immune system function $[45,46]$, decreased 2-5-fold on exposure to AgNP. Equally of concern could be the AgNP-associated decrease in two butyrogenic anaerobic species, Faecalibacterium prausnitzii (13-73\% reduction 
compared to control) and Roseburia faecalis (30-95\% reduction compared to control), which are suspected to be of benefit to their hosts due to their immune-modulatory and anti-inflammatory properties, and as well as their abilities to metabolize xenobiotics [47-50]. In regard to these butyrate-producing species, it is regrettable that this fatty acid (4:00) was below the detection limit (9:00) of our GC, and thus we could not further explore the influence of AgNPs on this particular fatty acid signature. However, the positive correlation $(r=0.76)$ between total fatty acid response and bacterial phylogenetic composition, and the significant decrease in some of the beneficial microbes in MET-1, together indicate a potential deleterious impact of AgNP on the human intestinal microbiota.

Although interest in host-microbiota interactions and their health implications has been growing [51,52], the influence of AgNPs on the intestinal microbiota, except for a study in Drosophila [17], appears to be unknown. We do not yet fully understand the mechanisms of toxicity of AgNPs towards other microbial communities, however, many groups have suggested that deleterious effects are due to the release of silver ion $\left(\mathrm{Ag}^{+}\right)$from AgNPs [53-56]. The AgNPs used here (PVP-coated with a zeta potential of $-25 \mathrm{mV}$ ) makes the release of substantial $\mathrm{Ag}^{+}$unlikely ( $1.8 \%$ of the total $\mathrm{Ag}$ was calculated in a previous studies $[6,7,57])$, with minimal losses in total concentration even over a 21 day exposure period [58]. Thus, toxicity was unlikely due to ionic silver, and this suggestion is supported by the differences seen after $\mathrm{AgNP}$ and $\mathrm{AgCl}$ treatments (e.g. the MDS analyses of $\mathrm{AgCl}$ and AgNP treatments). Others have attributed NP toxicity to the capping agent used [59], but here we saw no significant differences in PVPtreatment groups with any of the analyses. Another popular explanation is that AgNP exerts toxicity by forming reactive oxygen species (ROS) through the interaction of AgNPs with living bacterial cells [60]. Most obligate anaerobic bacteria lack ROS-scavenging enzymes since their action generates molecular oxygen, which in turn, damages enzymes required for metabolic activity [61]. Thus we speculate that the MET1 consortium might be particularly susceptible to NP-facilitated ROS production. Regardless of the mechanisms and/or mode of action, however, all three NP-toxicity profiles, including respiration, FAME, and the two DNA analyses, were distinct from those obtained with $\mathrm{AgCl}$ in the present study. Importantly, these experiments have now demonstrated the use of a robust and consistent model system for the testing of consumer product NPs on the human microbiota. In summary, our results clearly indicate that notwithstanding the impact of silver, a component of the impact of silver was nano size-dependent, and therefore AgNP ingestion, either deliberate or inadvertent, could have a negative impact on our intestinal microbiota. The subsequent influence of this on our overall health is presently unknown.

\section{Acknowledgement}

We thank Dr. G. Cairns (Analytical Services Lab, Queen's University), Dr. K. Jarrell (Queen's University), Dr. M. E. Hoque (Trent University), and Ms. M. Hay (Keystone Labs Inc.), for assistance with the gas chromatography, encouragement, as well as silver and fatty acid analyses, respectively. This research was supported by an NSERC Discovery grant to VKW and SEAMO (Southeastern Ontario Academic Medical Organization) grant to EOP

\section{References}

1. Nel A, Xia T, Mädler L, Li N (2006) Toxic potential of materials at the nanolevel. Science 311: 622-627.

2. Lok CN, Ho CM, Chen R, He Q Y, Yu WY, et al. (2007) Silver nanoparticles: Partial oxidation and antibacterial activities. J Biol Inorg Chem 12: 527-534.

3. Sotiriou GA, Pratsinis SE (2010) Antibacterial activity of nanosilver ions and particles. Environ Sci Technol 44: 5649-5654.

\section{4. http://www.nanotechproject.org/cpi/}

5. Marambio-Jones C, Hoek EMV (2010) A review of the antibacterial effects of silver nanomaterials and potential implications for human health and the environment. J Nanopart Res 12: 1531-1551.

6. Das P, Xenopoulos MA, Williams CJ, Hoque ME, Metcalfe CD (2012) Effects of silver nanoparticles on bacterial activity in natural waters. Environ Toxicol Chem 31: 122-130.

7. Das P, Williams CJ, Fulthorpe RR, Hoque ME, Metcalfe CD, et al. (2012) Changes in bacterial community structure after exposure to silver nanoparticles in natural waters. Environ Sci Technol 46: 9120-9128.

8. Kumar N, Palmer GR, Shah V, Walker VK (2014) The effect of silver nanoparticles on seasonal change in arctic tundra bacterial and fungal assemblages. PLoS One 9: e99953.

9. Rajilić-Stojanović M, Smidt H, de Vos WM (2007) Diversity of the human gastrointestinal tract microbiota revisited. Environ Microbiol 9: 2125-2136.

10. Payne AN, Zihler A, Chassard C, Lacroix C (2012) Advances and perspectives in in vitro human gut fermentation modeling. Trends Biotechnol 30: 17-25.

11. Bäckhed F, Ley RE, Sonnenburg JL, Peterson DA, Gordon JI (2005) Hostbacterial mutualism in the human intestine. Science 307: 1915-1920.

12. Eckburg PB, Bik EM, Bernstein CN, Purdom E, Dethlefsen L, et al. (2005) Diversity of the human intestinal microbial flora. Science 308: 1635-1638.

13. O'Hara AM, Shanahan F (2006) The gut flora as a forgotten organ. EMBO Rep 7: 688-693.

14. Biagi E, Nylund L, Candela M, Ostan R, Bucci L, et al. (2010) Through ageing, and beyond: gut microbiota and inflammatory status in seniors and centenarians. PLoS One 5: e10667.

15. Petrof EO, Gloor GB, Vanner SJ, Weese SJ, Carter D, et al. (2013) Stoo substitute transplant therapy for the eradication of Clostridium difficile infection: 'RePOOPulating' the gut. Microbiome 1: 3

16. https://atrium.lib.uoguelph.ca/xmlui/bitstream/handle/10214/7149/McDonald Julie_201305_\%20PhD.pdf?sequence=1

17. Han X, Geller B, Moniz K, Das P, Chippindale AK, et al. (2014) Monitoring the developmental impact of copper and silver nanoparticle exposure in Drosophila and their microbiomes. Sci Total Environ 487: 822-829.

18. White JM, Powell AM, Brady K, Russell-Jones R (2003) Severe generalized argyria secondary to ingestion of colloidal silver protein. Clin Exp Dermatol 28 254-256.

19. Brandt D, Park B, Hoang M, Jacobe HT (2005) Argyria secondary to ingestion of homemade silver solution. J Am Acad Dermatol 53: S105-107.

20. McDonald JAK, Schroeter K, Fuentes S, Heikamp-Dejong I, Khursigara CM et al. (2013) Evaluation of microbial community reproducibility, stability and composition in a human distal gut chemostat model. J Microbiol Methods 95: 167-174.

21. Choi O, Deng KK, Kim NJ, Ross L Jr, Surampalli RY, et al. (2008) The inhibitory effects of silver nanoparticles, silver ions, and silver chloride colloids on microbial growth. Water Res 42: 3066-3074.

22. Li WR, Xie XB, Shi QS, Zeng HY, Ou-Yang YS, et al. (2010) Antibacterial activity and mechanism of silver nanoparticles on Escherichia coli. Appl Microbio Biotechnol 85: 1115-1122.

23. Lu Z, Rong K, Li J, Yang H, Chen R (2013) Size-dependent antibacterial activities of silver nanoparticles against oral anaerobic pathogenic bacteria. $\mathrm{J}$ Mater Sci Mater Med 24: 1465-1471.

24. Sahakian AB, Jee SR, Pimentel M (2010) Methane and the gastrointestina tract. Dig Dis Sci 55: 2135-2143.

25. Haase ARB, Luch A, Dawson KA, Lynch I (2013) Mechanisms of silver nanoparticle release, transformation and toxicity: a critical review of current knowledge and recommendations for future studies and applications. Materials 6: 2295-2350.

26. Sasser M (1990) Identification of bacteria by gas chromatography of cellular fatty acids.

27. Bartelt-Ryser J, Joshi J, Schmid B, Brandi H, Balser T (2005) Soil feedbacks of plant diversity on soil microbial communities and subsequent plant growth Perspectives in Plant Ecology, Evol \& Systematics 7: 27-49. 
Citation: Das P, McDonald JAK, Petrof EO, Allen-Vercoe E, Walker VK (2014) Nanosilver-Mediated Change in Human Intestinal Microbiota. J Nanomed Nanotechnol 5: 235. doi: 10.4172/2157-7439.1000235

28. Muyzer G, de Waal DC, Uitterlinden AG (1993) Profiling of complex microbial populations by denaturing gradient gel electrophoresis analysis of polymerase chain reaction-amplified genes coding for $16 \mathrm{~S}$ rRNA. Appl Environ Microbio 59: $695-700$

29. Dowd SE, Callaway TR, Wolcott RD, Sun Y, McKeehan T, et al. (2008) Evaluation of the bacterial diversity in the feces of cattle using $16 \mathrm{~S}$ rDNA bacterial tagencoded FLX amplicon pyrosequencing (bTEFAP). BMC Microbiol 8: 125.

30. Gontcharova V, Youn E, Wolcott RD, Hollister EB, Gentry TJ, et al. (2010) Black Box Chimera Check (B2C2): a Windows-Based Software for Batch Depletion of Chimeras from Bacterial 16S rRNA Gene Datasets. Open Microbiol J 4: 47-52.

31. Bailey MT, Dowd SE, Parry NM, Galley JD, Schauer DB, et al. (2010) Stressor exposure disrupts commensal microbial populations in the intestines and leads to increased colonization by Citrobacter rodentium. Infect Immunol 78: 15091519.

32. Dowd SE, Zaragoza J, Rodriguez JR, Oliver MJ, Payton PR (2005) Windows NET Network Distributed Basic Local Alignment Search Toolkit (W.NDBLAST). BMC Bioinformatics 6: 93.

33. Cole JR, Wang Q, Cardenas E, Fish J, Chai B, et al. (2009) The Ribosomal Database Project: improved alignments and new tools for rRNA analysis. Nucleic Acids Res 37: D141-145.

34. Nyberg L, Turco RF, Nies L (2008) Assessing the impact of nanomaterials on anaerobic microbial communities. Environ Sci Technol 42: 1938-1943.

35. Roediger WE (2008) Review article: nitric oxide from dysbiotic bacteria respiration of nitrate in the pathogenesis and as a target for therapy of ulcerative colitis. Aliment Pharmacol Ther 27: 531-541.

36. Xie L, Ji C, Wang R, Zhou Q (2014) Microbial communities in anaerobic acidification-denitrification and methanogenesis process for cassava stillage treatment. Appl Mech Mat 522-524: 573-578.

37. Wong JWC, Li SWY, Wong MH (1995) Coal fly ash as a composting material for sewage sludge: effects on microbial activities. Environ Technol 16: 527-537.

38. Sinha R, Karan R, Sinha A, Khare SK (2011) Interaction and nanotoxic effect of $\mathrm{ZnO}$ and $\mathrm{Ag}$ nanoparticles on mesophilic and halophilic bacterial cells. Bioresour Technol 102: 1516-1520.

39. Suutari M, Laakso S (1994) Microbial fatty acids and thermal adaptation. Crit Rev Microbiol 20: 285-328.

40. Matsusue K, Ishii Y, Ariyoshi N, Oguri K (1997) A highly toxic PCB produces unusual changes in the fatty acid composition of rat liver. Toxicol Lett 91: 99104.

41. Heipieper HJ, Diefenbach R, Keweloh H (1992) Conversion of cis unsaturated fatty acids to trans, a possible mechanism for the protection of phenol-degrading Pseudomonas putida P8 from substrate toxicity. Appl Environ Microbiol 58 1847-1852.

42. Sakamoto M, Benno Y (2006) Reclassification of Bacteroides distasonis, Bacteroides goldsteinii and Bacteroides merdae as Parabacteroides distasonis gen. nov., comb. nov., Parabacteroides goldsteinii comb. nov. and Parabacteroides merdae comb. nov. Int J Syst Evol Microbiol 56: 1599-1605.

43. Silver S (2003) Bacterial silver resistance: molecular biology and uses and misuses of silver compounds. FEMS Microbiol Rev 27: 341-353.

44. Kumar N, Shah V, Walker VK (2012) Influence of a nanoparticle mixture on an arctic soil community. Environ Toxicol Chem 31: 131-135.

45. Wexler HM (2007) Bacteroides: the good, the bad, and the nitty-gritty. Clin Microbiol Rev 20: 593-621.

46. Coyne MJ, Reinap B, Lee MM, Comstock LE (2005) Human symbionts use a host-like pathway for surface fucosylation. Science 307: 1778-1781.
47. Segain JP, Raingeard de la Blétière $D$, Bourreille A, Leray V, Gervois N, et al. (2000) Butyrate inhibits inflammatory responses through NFkappaB inhibition: implications for Crohn's disease. Gut 47: 397-403.

48. Sokol H, Pigneur B, Watterlot L, Lakhdari O, Bermúdez-Humarán LG, et al. (2008) Faecalibacterium prausnitzii is an anti-inflammatory commensal bacterium identified by gut microbiota analysis of Crohn disease patients. Proc Natl Acad Sci U S A 105: 16731-16736.

49. Dabek M, McCrae SI, Stevens VJ, Duncan SH, Louis P (2008) Distribution of beta-glucosidase and beta-glucuronidase activity and of beta-glucuronidase gene gus in human colonic bacteria. FEMS Microbiol Ecol 66: 487-495.

50. Gloux K, Berteau O, El Oumami H, Béguet F, Leclerc M, et al. (2011) A metagenomic $\hat{l}^{2}$-glucuronidase uncovers a core adaptive function of the human intestinal microbiome. Proc Natl Acad Sci U S A 108 Suppl 1: 4539-4546.

51. Karlsson FH, Fåk F, Nookaew I, Tremaroli V, Fagerberg B, et al. (2012) Symptomatic atherosclerosis is associated with an altered gut metagenome. Nat Commun 3: 1245.

52. Arumugam M, Raes J, Pelletier E, Le Paslier D, Yamada T, et al. (2011) Enterotypes of the human gut microbiome. Nature 473: 174-180.

53. Yang X, Gondikas AP, Marinakos SM, Auffan M, Liu J, et al. (2012) Mechanism of silver nanoparticle toxicity is dependent on dissolved silver and surface coating in Caenorhabditis elegans. Environ Sci Technol 46: 1119-1127.

54. El Badawy AM, Silva RG, Morris B, Scheckel KG, Suidan MT, et al. (2011) Surface charge-dependent toxicity of silver nanoparticles. Environ Sci Technol 45: 283-287.

55. Lowry GV, Gregory KB, Apte SC, Lead JR (2011) Transformations of nanomaterials in the environment. Environ Sci Technol 46: 6893-6899.

56. Levard C, Hotze EM, Lowry GV, Brown GE Jr (2012) Environmental transformations of silver nanoparticles: impact on stability and toxicity. Environ Sci Technol 46: 6900-6914.

57. Das P, Metcalfe CD, Xenopoulos MA (2014) Interactive effects of silve nanoparticles and phosphorus on phytoplankton growth in natural waters. Environ Sci Technol 48: 4573-4580

58. Tejamaya M, Römer I, Merrifield RC, Lead JR (2012) Stability of citrate, PVP and PEG coated silver nanoparticles in ecotoxicology media. Environ Sci Technol 46: 7011-7017.

59. Suresh AK, Pelletier DA, Wang W, Morrell-Falvey JL, Gu B, et al. (2012) Cytotoxicity induced by engineered silver nanocrystallites is dependent on surface coatings and cell types. Langmuir 28: 2727-2735.

60. Xu H, Qu F, Xu H, Lai W, Andrew Wang Y, et al. (2012) Role of reactive oxygen species in the antibacterial mechanism of silver nanoparticles on Escherichia coli O157:H7. Biometals 25: 45-53.

61. Imlay JA (2002) How oxygen damages microbes: oxygen tolerance and obligate anaerobiosis. Adv Microb Physiol 46: 111-153. 